\title{
On-line dialysis with HPLC for the automated preparation and analysis of amino acids, sugars and organic acids in grape juice and wines*
}

\author{
C. Linget, C. Netter, D. Heems and E. Vérette**
}

Gilson S.A., BP. 45, 95400 Villiers-le-Bel, France

\begin{abstract}
A reproducible and high throughput technique is described for on-line clean-up and routine analysis of small molecules such as amino acids, sugars and organic acids in grape juice and wines. A fully automated sample processor (ASTED) provides an efficient way of preparing and cleaning-up raw liquid food samples. Following the usual preparation steps like dilution, addition of internal standards, mixing and derivatization, an on-line dialysis procedure is performed before HPLC analysis, to remove macromolecular and microparticulate interferents resulting from complex matrices. Eighteen amino acids, six organic acids, two sugars, ethanol and glycerol have been determined using two methods. Analytical data are provided both for a grape juice and a red wine.
\end{abstract}

Key words. sample preparation - amino acids - organic acids - sugars - wine

\section{Introduction}

Grape juice and wine quality assessment often requires HPLC of small molecules such as amino acids (AA), sugars and organic acids. Free AA in grape juice are impor- tant nitrogen sources for yeast during alcoholic fermentation, they also have a direct influence on aromatic composition of wines and have been successfully used to differentiate among wines according to grape variety or production area [1-4]. The simultaneous determination of sugars and organic

\footnotetext{
* Presented at In Vino Analytica Scientia, Bordeaux, 12-14 June, 1997. 


\section{Original articles}

acids concerns mainly the monitoring and the control of ripening and fermentation processes, up to the final product $[2,5,6]$. Indeed, some organic acids are regarded as indicators of ripeness or bacterial activities.

The limiting factor of this type of analysis is sample preparation. Efficient sample clean-up is essential for eliminating high-molecular-mass matrix interferents such as proteins, polysaccharides or condensed phenolic compounds like tannins, and microparticulates in order not to deteriorate the chromatographic results and the analytical column.

However, technological advances in sample preparation have been relatively few, when compared with those accomplished for HPLC. Until recently, the clean-up techniques employed prior to the HPLC determination of small analytes in grape juice, musts and wines have been essentially manual and off-line.

To overcome this problem, the ASTED sample processor (Fig. 1), with its on-line dialysis step, enables an efficient removal of macromolecular and microparticulate interferents (Fig. 2), followed by column switching for injection onto the HPLC system. The power of the technique may be further extended, according to the application, by performing a selective enrichment of the dialysate using a small trace enrichment cartridge before injection. The combination of on-line dialysis and trace enrichment provides an efficient and highly reproducible clean-up of raw liquid samples.

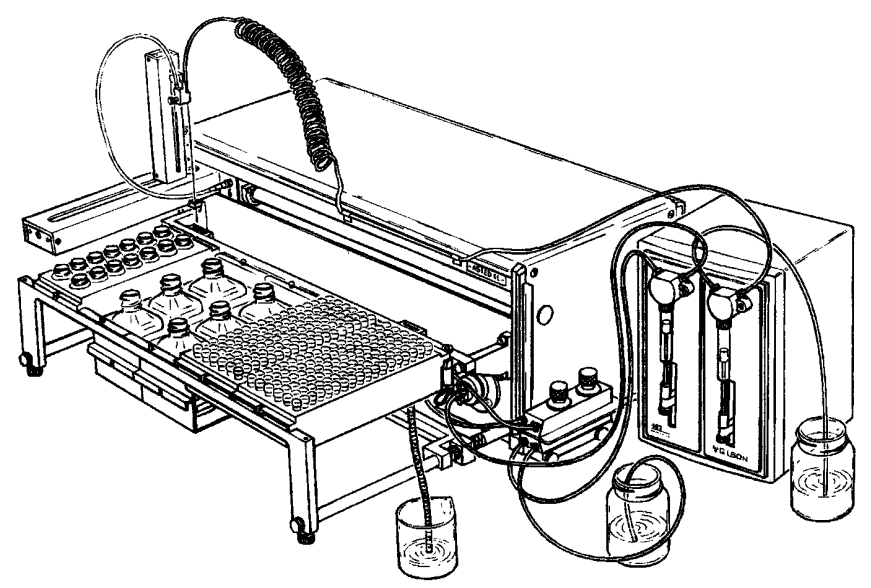

Fig. 1. ASTED sample processor.

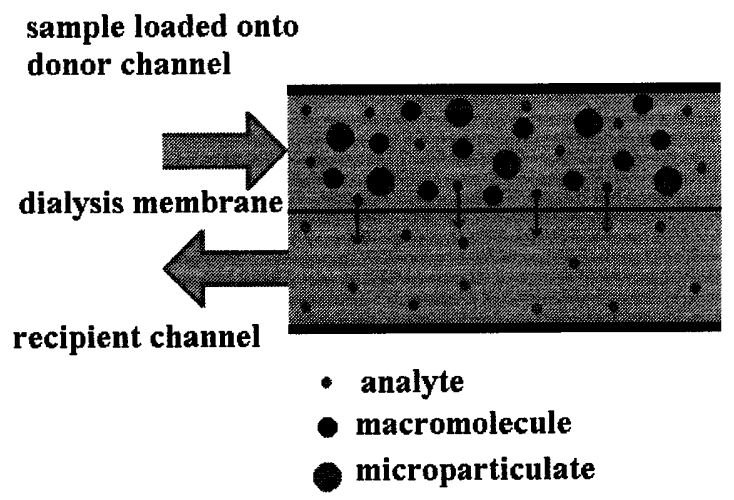

Fig. 2. Clean-up with on-line dialysis.
ASTED technology has been widely and successfully applied in many fields including food analysis for the determination of numerous small analytes [7-11].

This work describes an application of two methods previously developed for other food products, to determine: a - AA [11] and b - sugars, organic acids and related compounds like ethanol and glycerol [9], and specifically adapted here to grape juice and wine analysis. Method a uses a two step precolumn derivatization using 3-mercaptopropionic acid (3-MPA)/o-phthalaldehyde (OPA) for primary $\mathrm{AA}$, and 9-fluorenylmethyl chloroformate (FMOC) for secondary AA, before a reverse phase HPLC with a gradient elution and a fluorometric detection. OPA which is probably the most commonly used derivatizing reagent in reverse phase HPLC for the determination of primary AA, reacts efficiently in the presence of a thiol such as 3-MPA under alkaline conditions, to provide highly fluorescent derivatives; secondary AA which cannot react with OPA/3-MPA, are consequently derivatized with FMOC reagent before clean-up and analysis. Method b uses an ion exchange HPLC technique with an isocratic elution and a refractive index detection. Both methods perform an automatic cleanup using ASTED.

\section{Materials and methods}

\section{Materials}

Chemicals were of analytical grade and solvents of HPLC grade. The standards (AA, sugars, organic acids and glycerol) and the following reagents: OPA, 3-MPA, FMOC, iodoacetic acid (IDA), and sodium azide were obtained from Sigma (St Quentin Fallavier, France). Other reagents: sulphuric acid, $85 \%$ phosphoric acid, dibasic sodium phosphate anhydrous, $30 \%$ hydrochloric acid, 30\% sodium hydroxide, boric acid and dry acetone were obtained from MerckClevenot (Nogent-sur-Marne, France). Water, acetonitrile and tetrahydrofuran (THF) were obtained from J.T. Baker (Noisy-le-sec, France), methanol and ethanol from Carlo Erba (Val de Reuil, France). Samples were a commercial grape juice and a Cabernet Sauvignon wine from south of France.

\section{Preparation of reagents and solvents}

- Method a (amino acids analysis)

$100 \mathrm{mM}$ borate buffer: Boric acid (1.24 g) was dissolved in $200 \mathrm{ml}$ of water and adjusted to $\mathrm{pH} 10.4$ with sodium hydroxide. This buffer can be stored at room temperature for two weeks.

Internal standards / 3-MPA solution (reagent A): $25 \mu \mathrm{l}$ of norvaline (I.S.1) internal standard for primary AA and $50 \mu \mathrm{l}$ of thioproline (I.S.2) internal standard for secondary AA, both solutions at $2 \mathrm{mg} / \mathrm{ml}$ in water, were added to $9.875 \mathrm{ml}$ of borate buffer, then $50 \mu$ of 3 -MPA were added. This reagent must be prepared every week and kept at $4{ }^{\circ} \mathrm{C}$.

$100 \mathrm{mM}$ IDA solution (reagent B): IDA (0.208 g) was dissolved in $10 \mathrm{ml}$ borate buffer. This reagent can be kept at room temperature for two weeks.

$37.2 \mathrm{mM}$ OPA / $100 \mathrm{mM}$ 3-MPA (reagent C): OPA $(50 \mathrm{mg})$ was dissolved in $1 \mathrm{ml}$ of methanol, then borate 
buffer $(8.9 \mathrm{ml})$ was added, followed by 3-MPA $(100 \mu \mathrm{l})$. This reagent must be stored in amber glass vials at $4{ }^{\circ} \mathrm{C}$, and freshly prepared every week.

$5 \mathrm{mM}$ FMOC solution (reagent D): FMOC (13 mg) was dissolved in $10 \mathrm{ml}$ of dry acetone. This reagent must be freshly prepared every week and kept at $4{ }^{\circ} \mathrm{C}$.

HPLC mobile phase A: $10 \mathrm{mM}$ phosphate buffer was adjusted to $\mathrm{pH} 7.5$ with phosphoric acid and completed with $0.8 \%$ of THF.

HPLC mobile phase B: mobile phase A-methanol-acetonitrile $(20: 50: 30)$.

- Method b (sugars, organic acids, glycerol and ethanol analysis):

HPLC mobile phase: $0.0085 \mathrm{~N}$ sulphuric acid.

All HPLC mobile phases were filtered and degassed before use.

\section{Instrumentation}

Liquid samples were automatically prepared and cleaned-up using ASTED (Gilson, Villiers-le-Bel, France), consisting of a large capacity $X Y Z$ autosampler, a Model 402 dilutor (equipped with two 1-ml syringes), a flat-bed dialyser (cellulose acetate membrane with $15 \mathrm{kDa}$ mol. wt. cut-off, $100 \mu \mathrm{l}$ donor channel, $175 \mu \mathrm{l}$ recipient channel) and two sixport Model 7010 valves (Rheodyne, Berkeley CA, USA), one of which was fitted with a $20 \mu$ injection loop. For AA analysis, samples and reagents racks were thermostated at $4{ }^{\circ} \mathrm{C}$ using a Model 832 temperature regulator. Control was performed from a keypad using 722 (version 2.0) software.

The ASTED was coupled to an HPLC system (Gilson) as follows:

\section{- Method a}

Two Model 306 pumps with 5SC pump heads, a Model 805 manometric module, and a Model 811C mixer module for gradient elution, a Model 831 temperature regulator, a Model 122 fluorometer, and UniPoint (version 1.4) System Software for HPLC control and data handling. Derivatized AA were analyzed on a Hypersil BDS C18 column $(3 \mu \mathrm{m}$; $150 \times 4.6 \mathrm{~mm}$ ) from Hypersil (Runcorn, UK).

\section{- Method b}

One Model 306 pump fitted with a 5SC pump head for isocratic elution, a Model 805 manometric module, a Model 831 temperature regulator, a Model 133 refractive index detector, and UniPoint (version 1.4) System Software for HPLC control and data handling. The simultaneous determination of sugars, organic acids, ethanol and glycerol was performed on an Ion-300 column $(300 \times 7.8 \mathrm{~mm})$ from Interaction Chromatography (San Jose CA, USA).

\section{Methodology}

The procedures for method $\mathbf{a}$ and $\mathbf{b}$ are as follows:

\section{- Method a}

After performing an automatic dilution step, ASTED automated the following derivatization: sample $(20 \mu \mathrm{l})$ was added to $50 \mu \mathrm{l}$ of reagent $\mathrm{A}$, then reagent $\mathrm{B}(50 \mu \mathrm{l})$ was added to the sample-reagent A mixture, followed by $20 \mu \mathrm{l}$ of reagent $\mathrm{C}$ (OPA/3-MPA), and finally $10 \mu \mathrm{l}$ of reagent $\mathrm{D}$ (FMOC). After each reagent addition, two mixing cycles were performed. The reaction mixture was then cleaned up by on-line dialysis over $1.5 \mathrm{~min}$. Both the donor and recipient channels of the dialyser, containing aqueous $0.05 \%$ sodium azide solution to avoid contamination resulting from bacterial growth, were held in the static mode. The dialysate was then injected onto the HPLC system, and the donor channel was regenerated with $1 \mathrm{ml}$ of acetonitrile, followed by $4 \mathrm{ml}$ of water, and the recipient channel was regenerated with $5 \mathrm{ml}$ of water.

A simple binary gradient elution was performed from $0 \%$ to $70 \%$ of mobile phase B over 24 minutes, at $1.2 \mathrm{ml} / \mathrm{min}$, the column was then regenerated with $100 \%$ mobile phase $\mathrm{B}$ during 5 minutes and before returning to initial conditions. The column was thermostated at $40{ }^{\circ} \mathrm{C}$. OPA / 3-MPA derivatives were detected by the programmable fluorometer with excitation (Ex) and emission (Em) wavelengths set at $335 \mathrm{~nm}$ and $440 \mathrm{~nm}$ respectively; the FMOC derivatives were detected at Ex $260 \mathrm{~nm}$ and Em $315 \mathrm{~nm}$; the wavelength change occurred at $18.50 \mathrm{~min}$.

ASTED processed samples simultaneously with HPLC. The analysis time between injections was 38 min including the preparation time $(15 \mathrm{~min})$ and the reconditioning of the column.

\section{- Method b}

After performing an automatic dilution step with ASTED, adding an internal standard (propionic acid) and proceeding a mixing step, four fractions of samples $(50 \mu \mathrm{l}$ each) were successively loaded onto the donor channel of the dialyser in pulsed mode, the wait time was 2 minutes to perform dialysis. Both the donor and recipient channels of the dialyser contained aqueous $0.05 \%$ sodium azide solution. The dialysate was then injected onto the HPLC system, and the donor and recipient channels were regenerated with $5 \mathrm{ml}$ of water.

An isocratic elution was performed at $0.4 \mathrm{ml} / \mathrm{min}$. The column was thermostated at $65{ }^{\circ} \mathrm{C}$. Sugars, organic acids and related compounds were detected by a refractive index detector set at $0.03 \times 10^{-4}$ RIUFS. Sample processing and HPLC were performed contiguously: as one sample was being analyzed, the next was being prepared, which resulted in a complete run time of 35 minutes per sample.

\section{Results and discussion}

Chromatographic and sample preparation optimization, and validation on analytical standards have been previously developed $[9,11]$. Therefore, the present work focuses on the applicability of the methods in the analysis of vine products, and specific results obtained from a commercial grape juice and red wine.

\section{Amino acids analysis}

Among the 25 AA that the method can separate with standard solutions [11], 18 AA have been identified in the grape juice and red wine samples (Figs. 3 and 4). Limits of detection (LOD) of the $25 \mathrm{AA}$ at a signal-to-noise ratio of 3 were between $23 \mu \mathrm{g} / \mathrm{l}$ (glycine) and $278 \mu \mathrm{g} / \mathrm{l}$ (ornithine). In accordance with a previous work [3], cysteine, methionine and 


\section{Original articles}

tryptophan were not present, however ornithine which has been determined in the range of $5.3-31 \mathrm{mg} / \mathrm{l}$ from seven french red wines, was not detected in the Cabernet Sauvignon sample. The principal AA concentrations with their RSD are reported in table I. Results between grape juice and red wine cannot be directly compared because their origins are different, and some AA are partially or

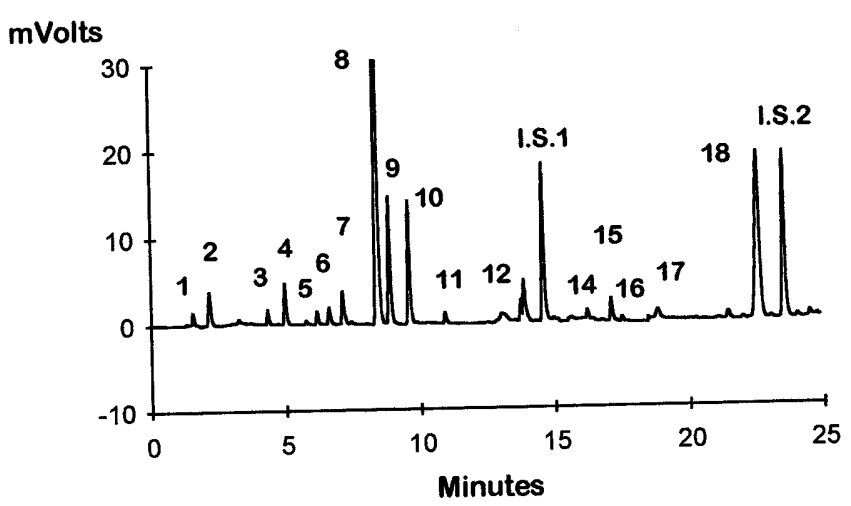

Fig. 3. HPLC analysis of amino acids derivatives in grape juice. 1: ASP, 2: GLU, 3: ASN, 4: SER, 5: HIS, 6: GLY, 7: THR, 8: ARG, 9: ALA, 10: GABA, 11: TYR, 12: VAL, 13: PHE, 14: ILE, 15: LEU, 16: LYS, 17: HPRO, 18: PRO.

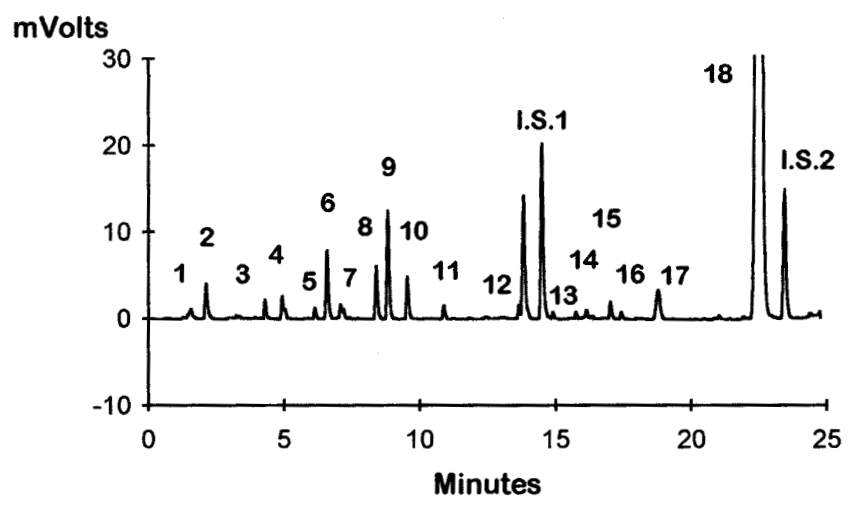

Fig. 4. HPLC analysis of amino acids derivatives in Cabernet Sauvignon red wine.

Table I. Principal amino acid content of grape juice and Cabernet Sauvignon red wine with their corresponding RSD $(n=10)$.

\begin{tabular}{lccccc}
\hline \multirow{2}{*}{$\begin{array}{l}\text { Peak } \\
N^{\circ}\end{array}$} & \multirow{2}{*}{ AA } & \multicolumn{2}{c}{ Grape juice } & \multicolumn{2}{c}{ Red wine } \\
& & $\begin{array}{c}\text { Conc. } \\
(\mathrm{mg} / \mathrm{l})\end{array}$ & $\begin{array}{c}\text { RSD } \\
\text { Conc. }\end{array}$ & $\begin{array}{c}\text { RSD } \\
(\mathrm{mg} / \mathrm{l})\end{array}$ & $\%$ \\
\hline 1 & ASP & 37.7 & 2.2 & 12.1 & 2.5 \\
2 & GLU & 61.7 & 1.1 & 28.5 & 1.3 \\
4 & SER & 26.0 & 1.3 & 8.4 & 1.5 \\
5 & HIS & 24.3 & 3.4 & 7.9 & 3.2 \\
7 & THR & 30.1 & 1.4 & 4.7 & 2.2 \\
8 & ARG & 531 & 1.9 & 21.1 & 1.3 \\
9 & ALA & 73.5 & 1.1 & 26.4 & 1.4 \\
10 & GABA & 81.6 & 1.5 & 11.7 & 1.6 \\
18 & PRO & 230 & 2.0 & 1810 & 1.9 \\
\hline
\end{tabular}

totally metabolized by yeasts during fermentation; however it shows the great importance of some AA like proline and arginine. As expected, proline is the most abundant AA in wine [2-4]. In this sample, the proline concentration is very high $(1810 \mathrm{mg} / \mathrm{l})$, which represents more than $90 \%$ of the total free AA. Arginine which is one of the most abundant AA in grape $(531 \mathrm{mg} / \mathrm{l}$ in the juice) is an important nutrient for yeast and bacteria growth and therefore it is found in a much lower concentration in wine $(21.1 \mathrm{mg} / \mathrm{l})$. The high ratio proline/arginine for the wine is in agreement with ratios usually found for Cabernet Sauvignon variety [4]. RSD for 10 real sample injections are between $1.1 \%$ and $3.4 \%$, which shows an excellent repeatability of the method.

\section{Sugars, organic acids, glycerol and ethanol analysis}

Glucose and fructose, 6 organic acids, glycerol and ethanol have been determined simultaneously. LOD at a signal-tonoise ratio of 3 , were between $0.07 \mathrm{~g} / \mathrm{l}$ and $0.11 \mathrm{~g} / \mathrm{l}$ for organic acids, and respectively $0.05 \mathrm{~g} / \mathrm{l}$ for glucose, $0.04 \mathrm{~g} / \mathrm{l}$ for fructose, $0.04 \mathrm{~g} / \mathrm{l}$ for glycerol and $0.10 \mathrm{~g} / \mathrm{l}$ for ethanol. Chromatograms for the analysis of grape juice and red wine are respectively given in figures 5 and 6 . Analyte

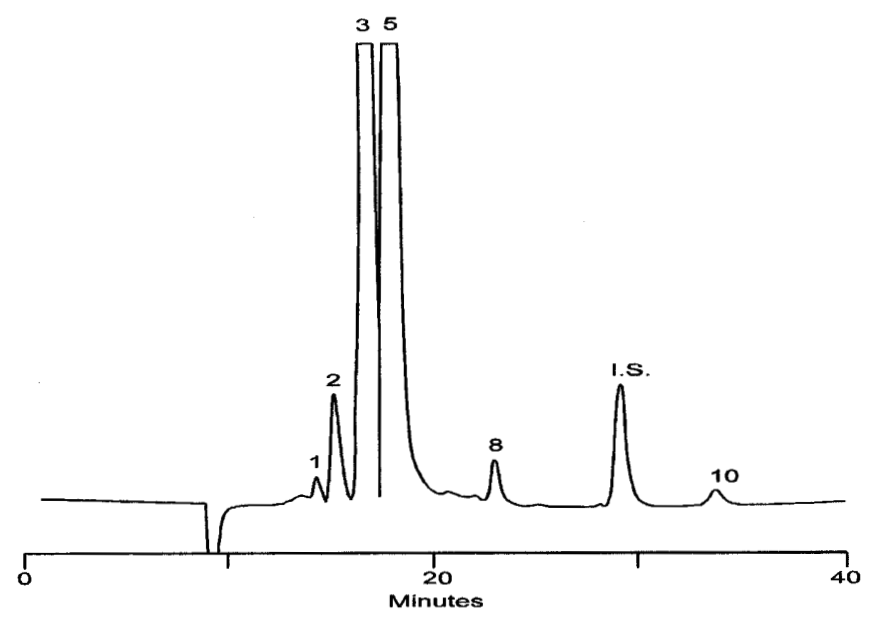

Fig. 5. HPLC analysis of sugars, organic acids and related compounds in grape juice.1: Citric acid, 2: Tartaric acid, 3: Glucose, 4: Malic acid, 5: Fructose, 6: Succinic acid, 7: Lactic acid, 8: Glycerol, 9: Acetic acid, 10: Ethanol.

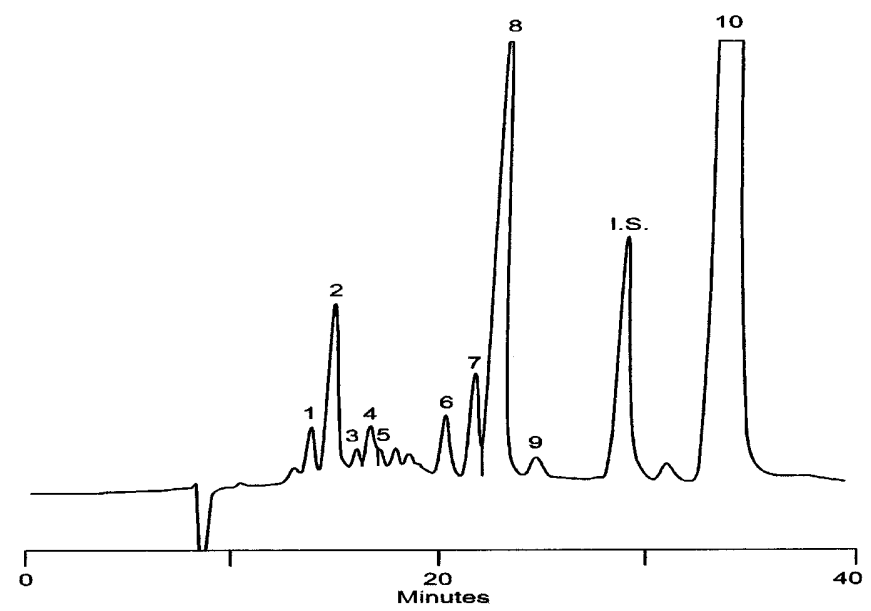

Fig. 6. HPLC analysis of sugars, organic acids and related compounds in Cabernet Sauvignon red wine. 
concentrations and their RSD are reported in table II, RSD were between $1.0 \%$ and $8.0 \%$. The natural organic acids in grape juice are tartaric, citric and malic acid. Malic acid could not be determined in the juice because of its low level compared to the high levels of the neighbouring sugars. This problem can be solved by lowering the column temperature, which results in a displacement of the malic acid out from the pair of sugars, but also in an important increase of the HPLC run time. Another solution can be to use a UV detector with the wavelength set at $210 \mathrm{~nm}$, which gives a much lower signal for sugars.

During fermentation, sugars are transformed into ethanol by yeasts with production of secondary constituents like succinic acid and glycerol. After alcoholic fermentation, red wines usually undergo the malo-lactic fermentation, in which malic acid is transformed into less acidic and more stable lactic acid. A slight oxidation of the wine may also result in the formation of acetic acid (peak 9 in Fig. 6).

No noticeable changes were observed in the chromatographic resolution nor in the dialysis method performance over 100 unattended injections of the red wine.

Table II. Sugar, organic acid and related compound content of grape juice and Cabernet Sauvignon red wine with their corresponding $\operatorname{RSD}(n=5)$.

\begin{tabular}{lcccc}
\hline Analyte & \multicolumn{2}{c}{ Grape juice } & \multicolumn{2}{c}{ Red wine } \\
& $\begin{array}{c}\text { Conc. } \\
(g / l)\end{array}$ & $\begin{array}{c}\text { RSD } \\
\%\end{array}$ & $\begin{array}{c}\text { Conc. } \\
(g / l)\end{array}$ & $\begin{array}{c}\text { RSD } \\
\%\end{array}$ \\
\hline Citric & 0.44 & 2.9 & 0.45 & 4.0 \\
Tartaric & 2.22 & 2.9 & 1.99 & 3.4 \\
Glucose & 44.10 & 1.4 & 0.15 & 4.6 \\
Malic & n.d. & - & 0.46 & 8.0 \\
Fructose & 39.10 & 1.2 & n.d. & - \\
Succinic & - & - & 0.70 & 1.6 \\
Lactic & - & - & 1.91 & 1.4 \\
Glycerol & 0.90 & 3.0 & 6.60 & 1.0 \\
Acetic & - & - & 0.51 & 3.2 \\
Ethanol & 2.20 & 7.8 & 122.0 & 2.2 \\
\hline
\end{tabular}

n.d. : not determined.

\section{Conclusion}

This work benefits from the advantages of on-line sample preparation in the HPLC analysis of small analytes such as amino acids, sugars and organic acids in liquid samples like grape juice, musts and wines. Raw samples were automatically prepared, cleaned-up by dialysis and injected directly into an HPLC system with satisfactory repeatability and sensitivity. The technique is perfectly suitable for the removal of macromolecular and microparticulate matrix interferents including insoluble FMOC in excess from secondary AA derivatization. This technique ideally replaces conventional off-line sample preparation procedures and results in more robust routine HPLC analyses.

Moreover it can be easily adapted with minimal method development, to the analysis of other small molecules such as biogenic amines, polyaromatic hydrocarbons, pesticides..., and applied to other types of samples from grape and wine industry by-products.

\section{References}

1. White, J. A.; Hart R. J. In: Food Analysis by HPLC; Nollet, L. M. L. Ed.; Marcel Dekker, New York, 1992, chpt 3.

2. Ough, C. S. In: Modern methods of plant analysis - New series Vol.6: Wine analysis; Springer-Verlag, Berlin; 1988, pp. 92-146.

3. Lehtonen P. Am. J. Enol. Vitic. 1996, 47, 127-133.

4. Huang, Z.; Ough, C. S. Am. J. Enol. Vitic., 1991, 42, 261-267.

5. Scott, F. W. In: Food Analysis by HPLC; Nollet, L. M. L. Ed.; Marcel Dekker, New York, 1992, chpt 8.

6. Gomis, D. B. In: Food Analysis by HPLC; Nollet, L. M. L. Ed.; Marcel Dekker, New York, 1992, chpt 11.

7. Vérillon, F.; Qian, F. Analusis 1991, 19, 271-277.

8. Van de Merbel, N. C.; Hageman, J. J.; Brinkman, U. A. T. J. Chromatogr. 1993, 634, 1-29.

9. Vérette, E.; Qian, F.; Mangani, F. J. Chromatogr. A 1995, 705, 195-203.

10. Cooper, J. D. H.; Turnell, D. C.; Green, B.; Demarais, D.; Rasquin, P. In: Sample Preparation for Biomedical and Environmental Analysis; Stenvenson, D.; Wilson, I.D. Eds.; Plenum Press, New York, 1994; pp 87-115.

11. Heems, D.; Luck, G.; Fraudeau, C.; Vérette, E. J. Chromatogr., in press. 